\title{
ABSOLUTELY CONTINUOUS INVARIANT MEASURES THAT ARE MAXIMAL
}

\author{
BY \\ W. BYERS ${ }^{1}$ AND A. BOYARSKY ${ }^{2}$
}

\begin{abstract}
Let $A$ be a certain irreducible 0-1 matrix and let $\tau$ denote the family of piecewise linear Markov maps on $[0,1]$ which are consistent with $A$. The main result of this paper characterizes those maps in $\tau$ whose (unique) absolutely continuous invariant measure is maximal, and proves that for "most" of the maps that are consistent with $A$, the absolutely continuous invariant measure is not maximal.
\end{abstract}

1. Introduction. In recent years there has been a great deal written on dynamical systems defined by maps $\tau$ of an interval into itself [1]. This stems from the realization that the simplest nonlinearities of such maps can introduce extreme dynamical complexity $[2,3]$. A conventional way of dealing mathematically with such "chaotic" dynamics is through the introduction of an invariant measure $\mu$ for $\tau$. When $\mu$ is ergodic, the Birkhoff Ergodic Theorem allows the computation of the frequency with which an orbit (iterates of a fixed starting point) hit a given set. For example,

$$
\lim _{N \rightarrow \infty} \frac{1}{N} \sum_{n=1}^{N} \chi_{A}\left(\tau^{n}(x)\right)=\mu(A)
$$

for almost every $x \in[0,1]$, with respect to $\mu$. However, there are usually many such ergodic measures available for a given transformation. For example, every measure supported on a finite periodic orbit is ergodic. But clearly such invariant measures cannot shed any new information about the dynamics of $\tau$. We are therefore led to the question: Which invariant measures of $\tau$ yield significant information about the dynamics of $\tau$ ?

There are two measures which appear prominently in the literature: measures absolutely continuous with respect to Lebesgue measure [4-8] and measures which maximize the measure theoretic entropy (maximal measures) [9-12]. In this paper we investigate the relationship between these two types of measures for a special class of Markov maps.

Received by the editors August 28, 1984.

1980 Mathematics Subject Classification. Primary 28D120; Secondary 26A18.

${ }^{1}$ The research of this author was supported by NSERC Grant A-5325 and an FCAC Grant from the Department of Education of Quebec.

${ }^{2}$ The research of this author was supported by NSERC Grant A-9072 and an FCAC Grant from the Department of Education of Quebec. 
In $\$ 2$ we translate the well-known results of Parry [23] to maps on an interval. This yields the following unsurprising result (Theorem 2): If $A$ is an irreducible 0-1 matrix with spectral radius $\lambda$, then the piecewise linear Markov map, $\tau$, consistent with $A$ that has constant slope $\lambda$ maximizes the measure theoretic entropy and the maximum is equal to $\log \lambda$.

In Theorem 3 of $\S 4$, we consider a certain irreducible 0-1 matrix $A$ and the family $\mathscr{T}$ of piecewise linear Markov maps, consistent with $A$, and we characterize those maps in $\mathscr{T}$ for which the (unique) absolutely continuous invariant measure is maximal. There are two main consequences of Theorem 3: (i) for certain irreducible 0-1 matrices $A$, the topological entropy can be achieved by families of maps with nonconstant slope, and (ii) in some cases, the attainment of the topological entropy by the absolutely continuous invariant measures implies the constant slope condition, which is the converse of Theorem 2 .

Our results are consistent with those obtained by Misiurewicz for Markov maps with strictly negative Schwarzian derivative (a case which is distinct from those we consider), from which it appears that the "absolutely continuous measure in general is not the measure with maximal entropy" [6]. In fact, from the remarks following Theorem 3, we can produce examples of $n-k$ parameter families of maps, $k=$ $1,2, \ldots, n-1$, where the maps whose absolutely continuous invariant measure is maximal is in a lower dimensional subspace. For all other transformations in the original family the absolutely continuous invariant measure and the maximal measure are different.

2. Piecewise linear Markov maps. Let $I=[0,1]$ and let $0=a_{0}<a_{1}<\cdots<a_{n}$ $=1$ be a partition of $I$ denoted by $\mathscr{P}$. A map $\tau: I \rightarrow I$ is called Markov if $\left.\tau\right|_{I_{i}}$ for $i=1,2, \ldots, n$, is a homeomorphism onto some interval $\left(a_{j(i)}, a_{k(i)}\right)$, where $I_{i}=$ $\left(a_{i-1}, a_{i}\right)$. If $\left.\tau\right|_{I_{i}}$ is linear for each $i$, then $\tau$ is referred to as a piecewise linear Markov map. Let $\mathscr{C}$ denote such maps.

Let $L_{1}$ be the space of integrable functions on $[0,1]$. Let $\tau: I \rightarrow I$ be nonsingular. We say that $f \in L_{1}$ is an invariant density under $\tau$ if

$$
\int_{A} f(x) d x=\int_{\tau^{-1}(A)} f(x) d x
$$

for all Lebesgue measurable sets $A$, where $\tau^{-1}(A)=\{x \in I: \tau(x) \in A\}$. It is well known [4] that the density $f$ is invariant under $\tau$ if and only if it is a fixed point of the Frobenius-Perron operator $P_{\tau}: L_{1} \rightarrow L_{1}$ defined by

$$
P_{\tau} f(x)=\frac{d}{d x} \int_{\tau^{-1}[0, x]} f(s) d s .
$$

If $\tau \in \mathscr{C}$, then it induces an $n \times n$ matrix $M=M_{\tau}$ defined as follows:

$$
m_{j l}=\delta_{j l} /\left|\tau_{j}^{\prime}\right|,
$$

where $\tau_{j}^{\prime}=d \tau /\left.d x\right|_{I_{j l}}$ and $\delta_{j l}=1$ if $I_{l} \subset \tau\left(I_{j}\right)$ and 0 otherwise. Thus, all nonzero entries of each row of $M$ are contiguous (no zero entries between nonzero entries) and equal, and the common value is $1 /\left|\tau_{j}^{\prime}\right|$. For a general partition $\mathscr{P}, M$ is not 
necessarily stochastic nor irreducible. In [14] it is shown that $M=P_{\tau}$ when $P_{\tau}$ is restricted to step functions on $\mathscr{P}$. Thus, if $\pi=\left(\pi_{1}, \ldots, \pi_{n}\right)$ is such a step function, then $\pi M=P_{\tau} \pi$. From this we see that if $M$ has a nonnegative left eigenvector associated with the eigenvalue 1 , then $\pi$, viewed as a function on $I$, is an invariant density under $\tau$.

Lemma 1 [15]. Let $\tau \in \mathscr{C}$. Then $M=M_{\tau}$ has 1 as its spectral radius. If $M$ is also irreducible, then the algebraic and geometric multiplicities of the eigenvalue 1 are also 1.

Hence $M$ always has fixed points, that is, if $\tau \in \mathscr{C}$, then there exists a step function $\pi$ which is a density invariant under $\tau$. In fact more is true.

Lemma 2 [16]. Let $\tau \in \mathscr{C}$ with respect to the partition $\mathscr{P}$, and assume $\left|\tau_{j}^{\prime}\right|>1$, $j=1, \ldots, n$. Then every density $f$ invariant under $\tau$ is a step function on $\mathscr{P}$.

In the sequal we shall need the following results:

Definition 1. Let $\mathscr{A}$ denote the set of $n \times n$ 0-1 matrices, where the nonzero entries of each row are contiguous (i.e. there are no zeros between ones).

Lemma 3. Let $A \in \mathscr{A}$ and let $D$ be a diagonal matrix with positive entries. If $A$ is irreducible and $B=D A$ has spectral radius equal to 1 , then there exists a map $\tau \in \mathscr{C}$ such that $M_{\tau}=D A$.

Proof. $B$ is irreducible since $A$ is. By the Perron-Frobenius Theorem [17] $B$ has a right eigenvector $\bar{u}=\left(u_{1}, \ldots, u_{n}\right)$ associated with the eigenvalue 1 , where $u_{i}>0$, $i=1, \ldots, n$. Furthermore, we can normalize $\bar{u}$ so that $\sum_{i=1}^{n} u_{i}=1$.

Partition [0,1] into subintervals $\left\{I_{i}\right\}_{i=1}^{n}$ such that the length of $I_{i}$ is $u_{k}$. Then define $\tau \in \mathscr{C}$ by setting

$$
\tau\left(I_{k}\right)=\bigcup_{j=1}^{n}\left\{I_{j}: a_{k j} \neq 0\right\}
$$

Since the nonzero entries of $A$ are contiguous we can take $\tau$ to be continuous on each interval $I_{k}, k=1,2, \ldots, n$. Now, the slope of $\left.\tau\right|_{I_{k}}$ is given by $\sum_{j=1}^{n} a_{k j} u_{j} / u_{k}$. But $B \bar{u}=\bar{u}$ means that

$$
u_{k}=\sum_{j=1}^{n} b_{k j} u_{j}=\sum_{j=1}^{n} d_{k} a_{k j} u_{j}=d_{k} \sum_{j=1}^{n} a_{k j} u_{j},
$$

where $D=\operatorname{diag}\left\{d_{1}, \ldots, d_{n}\right\}$. Thus

$$
d_{k}=u_{k} / \sum_{j=1}^{n} a_{k j} u_{j}=\text { reciprocal of slope of } k \text { th segment. }
$$

Therefore, $M_{\tau}=D A$.

Lemma 4. Let $A \in \mathscr{A}$ be irreducible and let it have maximal real eigenvalue $\lambda$. Then there exists a diagonal matrix with constant nonzero entries on the diagonal such that $D A$ is similar to a stochastic matrix. 
Proof. Since $A$ is irreducible there is a positive right eigenvector $z$ associated with $\lambda$. By Theorem 9.5.2 of [20], we know that $A=\lambda Z P Z^{-1}$, where $P$ is a stochastic matrix and $Z=\operatorname{diag}\left\{z_{1}, z_{2}, \ldots, z_{n}\right\}$. Let $D=\operatorname{diag}\{1 / \lambda, 1 / \lambda, \ldots, 1 / \lambda\}$. Then

$$
D A=\lambda D Z P Z^{-1}=Z P Z^{-1} \text {. }
$$

Corollary 1. $A \in \mathscr{A}$ be irreducible and have spectral radius $\lambda$. Then there exists a piecewise linear map $\tau:[0,1] \rightarrow[0,1]$ having constant slope $\lambda$ such that $M_{\tau}=A / \lambda$.

Let $\tau \in \mathscr{C}$ with respect to the partition $\mathscr{P}=\left\{I_{i}\right\}_{i=1}^{n}$ and let it induce the matrix $M=M_{\tau}=\left(m_{l j}\right), 1 \leqslant l \leqslant n, 1 \leqslant j \leqslant n$. Define the transition matrix $T=\left(t_{l j}\right)$ by

$$
t_{l j}=\frac{\lambda\left(I_{l} \cap \tau^{-1}\left(I_{j}\right)\right)}{\lambda\left(I_{l}\right)}=\frac{\lambda\left(I_{j}\right)}{\lambda\left(I_{l}\right)} m_{l j},
$$

where $\lambda$ is Lebesgue measure and $t_{l j}$ denotes the proportion of the interval $I_{l}$ which is mapped into $I_{j}$. Notice that $T$ has the same distribution of nonzero entries as $M$. It is also (row) stochastic.

Definition 2. The measure theoretic entropy of $\tau$ with respect to the measure $\mu$ invariant under $\tau$ is given by the expression [18, p. 91]:

$$
H(\tau, \mu)=-\sum_{j=1}^{n} \sum_{i=1}^{n} \mu_{i} t_{i j} \log t_{i j},
$$

where $\mu_{i}=\mu\left(I_{i}\right), i=1, \ldots, n$.

For the basic definition of topological entropy, $H(\tau)$, we refer the reader to [13, 19]. Its relation to measure entropy is through:

Lemma 5 [19, Theorem 8.6]. Let $\tau: I \rightarrow I$ be a continuous map. Then $H(\tau)=$ $\sup \{H(\tau, \mu): \mu$ is a probability measure invariant under $\tau\}$.

Definition 3. A measure $\mu$ such tht $H(\tau, \mu)=H(\tau)$ is referred to as a maximal measure.

Let $A \in \mathscr{A}$ be irreducible and have spectral radius $\lambda>1$. By Corollary 1 there exists a map $\tau \in \mathscr{C}$ with constant slope $\lambda>1$. Let $\tau$ be any map in $\mathscr{C}$ such that $M_{\tau}=D A$ for some diagonal matrix $D$, whose nonzero entries are greater than 1 in absolute value. We then say $\tau$ is consistent with $A$.

Let

$$
X_{A}=\left\{\left(x_{k}\right)_{k=0}^{\infty}: a_{x_{k} x_{k+1}}=1, k=0,1, \ldots\right\} \text {, }
$$

where $x_{k} \in\{1, \ldots, n\}$ for $k=0,1, \ldots$, and $X_{A}$ is the symbol space consisting of all sequences $\left(x_{k}\right)_{k=0}^{\infty}$ of symbols whose neighbouring pairs are allowed by the matrix $A$. Let $T: X_{A} \rightarrow X_{A}$ be the shift map. (For more details on symbolic dynamics see [19, Chapter 5].)

Proposition 1. Let $A \in \mathscr{A}$ be irreducible and have spectral radius $\lambda>1$. Let $\tau \in \mathscr{C}$ be an expanding map induced by $A$; for example $\tau$ is such that $M_{\tau}=A / \lambda$. Then there exists a continuous map $\psi: X_{A} \rightarrow I$ such that $\tau \circ \psi=\psi \circ T$, where $\psi$ is a homeomorphism from $X_{A}$ onto $I-C$, and $C$ is a countable set. 
Proof. Let the Markov partition of $I$ be given by $I_{1} \leqslant I_{2} \leqslant \cdots \leqslant I_{n}$. Then $\psi$ is defined by the formula

$$
\psi\left(\left(x_{n}\right)_{n=0}^{\infty}\right)=\bigcap_{n=0}^{\infty} \tau^{-n}\left(I_{x_{n}}\right) .
$$

This intersection is nonempty because $\tau$ is Markov and it consists of a single point because $\tau$ is expanding. It is not difficult to check that $\psi$ is a conjugacy, i.e. $\tau \circ \psi=\psi \circ T$, and that $\psi$ is a homeomorphism onto $I-C$, where $C$ is the set if inverse images of the end points of the intervals $I_{1}, \ldots, I_{n}$ (cf. Nitecki [21]).

We shall be using the following result of Parry's [22] as summarized in [23]:

TheOREM 1. If $\left(X_{A}, T\right)$ is regionally transitive, then there exists a unique normalized Borel invariant measure, $\mu$, of maximal entropy. Moreover, there also exists a normalized measure $p$ equivalent to $\mu$ such that $p(T C)=\beta p(C)$ for any cylinder set $C$ where $\log \beta=H(T)$.

Definition 4. $\left(X_{A}, T\right)$ is regionally transitive if for every pair $C, D$ of nonempty cylinder sets there exists an integer $n$ such that $C \cap T^{-n} D \neq 0$. This is implied by the condition [22] that

$$
C \neq \varnothing \Rightarrow \bigcup_{n=0}^{\infty} T^{n} C=X_{A} .
$$

In our case, $\tau$ is a Markov map induced by the 0-1 matrix $A$, which is irreducible. Thus for every pair $(i, j)$ there exists an $n$ such that $a_{i j}^{n} \neq \varnothing$, i.e. $\tau^{n}\left(I_{i}\right) \supseteq I_{j}$. Therefore, $\bigcup_{n=0}^{\infty} \tau_{n}\left(I_{i}\right)=[0,1]$. If $C=I_{a_{0}} \cap \tau^{-1}\left(I_{a_{1}}\right) \cap \cdots \cap \tau^{-k}\left(I_{a_{k}}\right) \neq \varnothing$, then there exists an interval $J \subseteq I_{a_{0}}$ such that $\tau^{t}(J) \subseteq I_{a_{t}}, t=0, \ldots, k-1$, and $\tau^{k}(J)=$ $I_{a_{k}}$, i.e., $J \subseteq C$ and $\bigcup_{n=0}^{\infty} \tau^{n K}(J) \supseteq \bigcup_{n=0}^{\infty} \tau^{n}\left(I_{a_{k}}\right)=[0,1]$. Thus,

$$
\bigcup_{n=0}^{\infty} \tau^{n}(C)=[0,1] \text {. }
$$

Therefore, the associated symbolic system $\left(X_{A}, T\right)$ is regionally transitive. Thus we obtain from Theorem 1:

Proposition 2. If the symbolic system $\left(X_{A}, T\right)$ is obtained from the map $\tau \in \mathscr{C}$ (as in Proposition 1), then it admits a unique maximal measure (the Parry measure).

THEOREM 2. Let $\tau \in \mathscr{C}$ be expanding and of constant slope (i.e. the absolute value of the slope is constant). Suppose that the 0-1 matrix $A$ which it induces is irreducible. Then there exists a unique Borel probability measure $\mu$, invariant under $\tau$, which maximizes entropy and it is equivalent to Lebesgue measure.

Proof. We have already commented that the associated system $\left(X_{A}, T\right)$ has a unique maximal measure; call it $\nu$. Define $\mu$ on $[0,1]$ by setting $\mu(B)=\nu\left(\psi^{-1}(B)\right)$, where $\psi$ is the map defined in Proposition 1 . Thus $\mu$ is an invariant probability measure for $\tau$ which maximizes entropy.

According to Theorem 1 there exists a normalized measure $p$ equivalent to $\nu$ such that $p(T C)=\beta p(c)$ for any cylinder set $C$. Defining $m(B)=p\left(\psi^{-1}(B)\right)$, we have a 
measure equivalent to $\mu$ on $[0,1]$ such that

$$
m(\tau(D))=p\left(\psi^{-1} \tau(D)\right)=p\left(T \psi^{-1}(D)\right)=\beta p\left(\psi^{-1}(D)\right)=\beta m(D) .
$$

Now suppose $D=I_{i}$ for some $i$. Then $\tau\left(I_{i}\right)=\bigcup_{j} a_{i j} I_{j}$, and so

$$
\beta m\left(I_{i}\right)=m\left(\tau\left(I_{i}\right)\right)=\sum_{j=1}^{n} a_{i j} m\left(I_{j}\right), \quad i=1, \ldots, n,
$$

i.e. the vector $\left(m\left(I_{i}\right), \ldots, m\left(I_{n}\right)\right)$ is a positive right eigenvector for $A$ with eigenvalue $\beta$. Now if $A$ is irreducible there is a unique eigenvalue $\beta$ with nonnegative eigenvector. The corresponding eigenspace is one dimensional (see proof to Theorem 9.2.1 in [20]) and therefore the numbers $\left(m\left(I_{1}\right), \ldots, m\left(I_{n}\right)\right)$ are uniquely determined and are in fact the lengths of the intervals $I_{1}, \ldots, I_{n}$. Since the lengths also satisfy the equation length $\left(\tau\left(I_{i}\right)\right)=\beta$ length $\left(I_{i}\right)$, where $\beta$ is the (constant) slope, it then follows that $m(\cdot)$ is in fact Lebesgue measure for all sets of the form $I_{a_{0}} \cap \tau^{-1}\left(I_{a_{1}}\right) \cap \cdots \cap$ $\tau^{-n}\left(I_{a_{n}}\right)$.

REMARKs. 1. Since $A$ is irreducible, $M_{\tau}$ is also irreducible. Hence, in view of Lemma 1, each $\tau$ consistent with $A$ admits a unique absolutely continuous measure $\eta$. Thus, the measure $\mu$ of Theorem 2 is equal to $\eta$, and the density of $\mu$ is the solution of $u M_{2}=u_{2}$.

2. Although Theorem 2 appears to be a known result, we were unable to find this formulation in the literature.

\section{Two examples.}

Example 1. Consider the $n \times n$ matrix $A$ defined by $a_{11}=1, a_{i, i+1}=1, i=$ $1,2, \ldots, n-1, a_{n, 1}=1$ and $a_{i j}=0$ elsewhere. Let $0<a_{1}<\cdots<a_{n-1}=1$ be a partition of $I$ and let $\tau: I \rightarrow I$ be the piecewise linear Markov map consistent with $A$. The matrix $M=M_{\tau}$ induced by $\tau$ is given by:

$$
\left(\begin{array}{ccccc}
a_{1} / a_{2} & a_{1} / a_{2} & 0 & \cdots & 0 \\
0 & 0 & \left(a_{2}-a_{1}\right) /\left(a_{3}-a_{2}\right) & \cdots & 0 \\
0 & 0 & \cdots & \cdots & \left(a_{n-1}-a_{n-2}\right) /\left(1-a_{n-1}\right) \\
\left(1-a_{n-1}\right) / a_{1} & 0 & 0 & \cdots & 0
\end{array}\right) .
$$

The unique normalized left eigenvector $v$ associated with the eigenvalue 1 and $M$ can easily be found and from it the probabilities of the $n$ intervals, given by

$$
\mu=\left(\frac{a_{2}}{n\left(a_{2}-a_{1}\right)+a_{1}}, p_{2}, p_{2}, \ldots, p_{2}\right),
$$

where $p_{2}=\left(a_{2}-a_{1}\right) /\left(n\left(a_{2}-a_{1}\right)+a_{1}\right)$. With the aid of the transition matrix for $\tau$ it is easy to show that

$$
H(\tau, \mu)=-\left[\frac{a_{2}}{n\left(a_{2}-a_{1}\right)+a_{1}}\left(\frac{a_{1}}{a_{2}} \log \frac{a_{1}}{a_{2}}+\frac{a_{2}-a_{1}}{a_{2}} \log \frac{a_{2}-a_{1}}{a_{2}}\right)\right] \equiv H\left(a_{1}, a_{2}\right) .
$$

$H\left(a_{1}, a_{2}\right)$ is a homogeneous function. Let $z=a_{1} / a_{2}$. Then define

$$
H(z)=-\left[\frac{1}{n(1-z)+z}(z \log z+(1-z) \log (1-z))\right] .
$$


Setting $(d / d z) H(z)=0$, we derive the equation

$$
z^{n}+z-1=0
$$

which has a unique positive root $\alpha$ between 0 and 1 for all values of $n$. It follows that $\beta=1 / \alpha>1$ is a root of

$$
\lambda^{n}-\lambda^{n-1}-1=0
$$

which is the characteristic equation of $A$. Now it is well known [19] that the topological entropy of $\tau$ (consistent with $A$ ) is given by $\log \rho$, where $\rho$ is the spectral radius of $A$. Thus $\rho$ is the positive real eigenvalue of $A$, i.e., $\rho=1 / \alpha$. Since we know by Theorem 2 that the entropy of the absolutely continuous invariant measure for $\tau$ (with constant slope) is the topological entropy, we obtain: given any partition $0<a_{1}<a_{2}<\cdots<a_{n-1}<1$ such that $a_{2} / a_{1}=\alpha$, then the (unique) absolutely continuous measure invariant under $\tau$ is maximal.

Thus we have shown that for this $(n-1)$ parameter family of maps there exists an $(n-2)$ dimensional subfamily such that for each of its members the (unique) absolutely continuous invariant measure is maximal. For all other transformations in the original family the absolute continuous invariant measure and the maximal measure are different.

ExAmple 2. Let $0=a_{0}<a_{1}<\cdots<a_{n}=1$ be any partition of $I$. Define the piecewise linear, continuous Markov map $\tau: I \rightarrow I$ by

$$
\begin{aligned}
& \tau\left(I_{i}\right)=I_{i+1}, \quad 1 \leqslant i \leqslant n, \\
& \tau\left(I_{n}\right)=I .
\end{aligned}
$$

Straightforward computations show that the (unique) absolutely continuous measure invariant under $\tau$ has (normalized) density given by $\pi=\left(\pi_{1}, \pi_{2}, \ldots, \pi_{n}\right)$, where

$$
\pi_{i}=\left.\pi\right|_{I_{i}}=\frac{1}{\left(\sum_{i=1}^{n} a_{n}\right)} \frac{a_{i}}{\left(a_{i}-a_{i-1}\right)} .
$$

Thus, the measure of the $i$ th subinterval, $I_{i}$, is given by $u_{i}=a_{i} / \sum_{i=1}^{n} a_{i}$. To compute the measure-theoretic entropy for $\tau$, we need to know only the last row in the transition matrix, since each of the other rows will have only a single entry equal to 1. Clearly

$$
t_{n, i}=\frac{\lambda\left[\tau^{-1}\left(I_{i}\right) \cap I_{n}\right]}{\lambda\left(I_{n}\right)}=a_{i}-a_{i-1} .
$$

Hence the measure-theoretic entropy of $\tau$ for the given partition, denoted by $H(\tau, \bar{a})$, is given by

$$
\begin{aligned}
H(\tau, \bar{a}) & =-\sum_{i=1}^{n} \frac{1}{S_{n}}\left(a_{i}-a_{i-1}\right) \ln \left(a_{i}-a_{i-1}\right) \\
& =\ln \prod_{i=1}^{n}\left\{\left(a_{i}-a_{i-1}\right)^{-\left(a_{i}-a_{i-1}\right) / S_{n}}\right\},
\end{aligned}
$$


where $S_{n}=\sum_{i=1}^{n} a_{i}$. On differentiating $H(\tau, \bar{a})$ with respect to $a_{1}, a_{2}, \ldots, a_{n}$ and setting each term equal to 0 , we derive the unique solution

$$
\frac{a_{1}}{a_{2}-a_{1}}=\frac{a_{3}-a_{2}}{a_{2}-a_{1}}=\cdots=\frac{a_{n-1}-a_{n-2}}{a_{n-2}-a_{n-1}}=\frac{a_{n}-a_{n-1}}{a_{n}},
$$

i.e., $\tau$ has constant slope. Clearly $\tau$ is unique.

In the above two examples, we have displayed two extremely different results. In the first example, we showed that for certain irreducible 0-1 matrices $A$, the space of piecewise linear Markov maps $\tau$ consistent with $A$ contains an $(n-2)$ dimensional subspace of maps whose absolutely continuous invariant measures are maximal.

In the second example, we showed that for certain $A$ the subspace of maps whose absolutely continuous invariant measures are maximal is zero dimensional, that is, it consists of one map only, the map with constant slope.

These opposite types of behavior are unified in the following section.

4. The main theorem. Define the class of matrices $\mathscr{A}_{0} \subset \mathscr{A}$ as follows: $A=\left(a_{i j}\right) \in$ $\mathscr{A}_{0}$ if (i) $A$ is irreducible and (ii) there are integers $p$ and $q, 1 \leqslant p \leqslant q \leqslant n$, such that every row of $A$ either consists of a block of 1 's, $a_{i j}=1$ if and only if $j=p, \ldots, q$, or else the row contains a unique nonzero element; $a_{i j}=1$. Let this if $j=\sigma(i)$. [We assume that there is at least one row of each type.]

Given such a matrix $A$, let $0=a_{0}<a_{1}<\cdots<a_{n-1}<a_{n}=1$ be a partition of $[0,1]$ and $\tau$ a piecewise linear Markov map on $[0,1]$ consistent with this matrix. Let $I_{i}=\left[a_{i-1}, a_{i}\right], i=1, \ldots, n$, and $J=\left[a_{p-1}, a_{q}\right]$.

THEOREM 3. The (unique) absolutely continuous invariant measure for $\tau$ is maximal if and only if $\tau$ has (constant) slope $\lambda$ on all the intervals $I_{i} \subset J$, where $\lambda$ is the spectral radius of $A$.

Proof. Let $S=\{1,2, \ldots, n\}$ and let $S_{j}=\{i \in S: j$ is the smallest integer for which $\left.\tau^{j}\left(I_{i}\right)=J\right\}, j=1, \ldots, k$. The sets $\left\{S_{j}\right\}$ are pairwise disjoint. Moreover, if some interval $I_{a}$ never mapped onto $J$ we could find in its orbit under $\tau$ a cycle with $\tau^{i}$ mapping $I_{b}$ homeomorphically onto itself for some $b \in S$ and $i \in \mathbf{Z}^{+}$. Since $A$ is irreducible this cycle would have to include every interval $I_{j}, j=1, \ldots, n$. Therefore every subinterval eventually maps onto all of $J$ and $\bigcup_{j=1}^{k} S_{j}=S$.

Let $\alpha_{i}=a_{i}-a_{i-1}$ so that $\sum_{i=1}^{n} \alpha_{i}=1$. The absolutely continuous invariant density for $\tau$ is given by the left eigenvector (of the eigenvalue 1) of the matrix $M_{\tau}$ which is given by

$$
m_{i j}= \begin{cases}\alpha_{i} a_{i j} / \alpha & \text { for } p \leqslant i \leqslant q, \\ \alpha_{i} a_{i j} / \alpha_{\sigma(i)} & \text { for } i<p \text { or } i>q\end{cases}
$$

where $\alpha=a_{q}-a_{p-1}=\lambda(J)$. The left eigenvector is the solution of the equations:

$$
\sum_{i=1}^{n} \pi_{i} m_{i a}=\pi_{a}, \quad a=1, \ldots, n
$$


Set $d=\sum\left\{\pi_{i} \alpha_{i} / \alpha: i \in S_{1}\right\}$. Then the above equations become

$$
\pi_{a}= \begin{cases}\sum\left\{\alpha_{i} \pi_{i} / \alpha_{a}: \sigma(i)=a\right\} & \text { for } a<p \text { or } a>q, \\ \alpha+\sum\left\{\alpha_{i} \pi_{i} / \alpha_{a}: \alpha(i)=a\right\} & \text { for } p \leqslant a \leqslant q,\end{cases}
$$

where the summation is taken to be zero if there is no value of $i$ for which $\sigma(i)=a$.

Setting $d=1$ we obtain the associated probability vector with components

$$
p_{a}=\alpha_{a} \pi_{a}= \begin{cases}\sum\left\{\alpha_{i} \pi_{i}: \sigma(i)=a\right\} & \text { for } a<p \text { or } a>q, \\ \alpha_{a}+\sum\left\{\alpha_{i} \pi_{i}: \sigma(i)=a\right\} & \text { for } p \leqslant a \leqslant q .\end{cases}
$$

Suppose $\sigma(i)=a$. Then

$$
\alpha_{i} \pi_{i}= \begin{cases}\sum\left\{\alpha_{j} \pi_{j}: \sigma(j)=i\right\} & \text { for } i<p \text { or } i>q \\ \alpha_{i}+\sum\left\{\alpha_{j} \pi_{j}: \sigma(j)=i\right\} & \text { for } p \leqslant i \leqslant q .\end{cases}
$$

Thus if $p \leqslant a \leqslant q$

$$
p_{a}=\alpha_{a}+\sum\left\{\alpha_{i}: p \leqslant i \leqslant q \text { and } \sigma(i)=a\right\}+\sum\left\{\alpha_{j} \pi_{j}: \sigma^{2}(j)=a\right\} .
$$

Continuing this process for (at most) $k$-steps, we derive the formula

$$
p_{a}=\alpha_{a}+\sum\left\{\alpha_{i}: p \leqslant i \leqslant q, \sigma(i)=a\right\}+\cdots+\sum\left\{\alpha_{i}: p \leqslant i \leqslant q, \sigma^{k}(i)=a\right\}
$$

which we write as

$$
p_{a}=\sum_{j=0}^{k} \sum_{i}\left\{\alpha_{i}: p \leqslant i \leqslant q, \sigma^{j}(i)=a\right\} .
$$

The same formula holds for $a<p$ or $a>q$ (of course here the term $\alpha_{a}$ corresponding to $j=0$ is missing).

Using this formula we can compute

$$
\begin{aligned}
c & =\sum_{a=1}^{n} p_{a}=\sum_{a=1}^{n} \sum_{j=0}^{k} \sum_{i}\left\{\alpha_{i}: p<i<q, \sigma^{j}(i)=a\right\} \\
& =\sum_{a=1}^{n} \sum_{j=1}^{k}\left\{j \alpha_{a}: p \leqslant a \leqslant q, a \in S_{j}\right\}
\end{aligned}
$$

since if $a \in S_{j}, p \leqslant a \leqslant q$, then $I_{a}$ maps homeomorphically onto exactly $j$ intervals under powers of $\tau$ before it maps onto $J$. Then the (normalized) invariant density of the absolutely continuous invariant measure for $\tau$ is given by $(1 / c)\left(p_{1}, \ldots, p_{n}\right)$ and the entropy of this measure is [19]:

$$
H(\tau)=H\left(\alpha_{1}, \ldots, \alpha_{n}\right)=-\sum_{i, j} \frac{p_{i}}{c} p_{i j} \log p_{i j},
$$

where $p_{i j}$ is the probability of going from the $i$ th to the $j$ th interval. Thus $p_{i j} \log p_{i j}=0$ for $i \notin S_{1}$ (since $p_{i j}$ either equals 0 or 1 ). For $i \in S_{1}$ we have

$$
p_{i j}= \begin{cases}\alpha_{j} / \alpha & \text { for } p \leqslant j \leqslant q \\ 0 & \text { for } j<p \text { or } j>q .\end{cases}
$$


Thus,

$$
H(\tau)=-\frac{1}{c}\left(\sum_{i \in S_{1}} p_{i}\right)\left(\sum_{i=p}^{q} \frac{\alpha_{i}}{\alpha} \log \frac{\alpha_{i}}{\alpha}\right)
$$

Now

$$
\begin{aligned}
\sum_{i \in S_{1}} p_{i} & =\sum\left\{\alpha_{j}: p \leqslant j \leqslant q \text { and } I_{j} \text { eventually maps onto } J\right\} \quad \text { (using (1)) } \\
& =\sum\left\{\alpha_{j}: p \leqslant j \leqslant q\right\}=\alpha
\end{aligned}
$$

Therefore

$$
H\left(\alpha_{1}, \ldots, \alpha_{n}\right)=-\frac{\alpha}{c} \sum_{i=p}^{q} \frac{\alpha_{i}}{\alpha} \log \frac{\alpha_{i}}{\alpha}=-\frac{1}{c}\left(\sum_{i=p}^{q} \alpha_{i} \log \frac{\alpha_{i}}{\alpha}\right)
$$

Now,

$$
\frac{\partial H}{\partial \alpha_{a}}=\left\{\begin{array}{l}
0 \quad \text { for } a<p \text { or } a>q \\
\frac{j}{c^{2}} \sum_{i=p}^{q} \alpha_{i} \log \frac{\alpha_{i}}{\alpha}-\frac{1}{c}\left(1+\log \frac{\alpha_{a}}{\alpha}-\sum_{i=p}^{q} \frac{\alpha_{i}}{\alpha}\right) \text { for } p \leqslant a \leqslant q, a \in S_{j} .
\end{array}\right.
$$

Thus using Lagrange multipliers on $H(\tau)-\lambda\left(\sum \alpha_{i}-1\right)$ yields the equations:

$$
j \sum_{i=p}^{q} \alpha_{i} \log \frac{\alpha_{i}}{\alpha}=c\left(1+\log \frac{\alpha_{a}}{\alpha}-\sum_{i=p}^{q} \frac{\alpha_{i}}{\alpha}\right) \text { for } p \leqslant a \leqslant q, a \in S_{j} .
$$

Thus if $a, b \in S_{j}, p \leqslant a, b \leqslant q$, and we can conclude that $\log \left(\alpha_{a} / \alpha\right)=\log \left(\alpha_{b} / \alpha\right)$ and so

$$
\alpha_{a}=\alpha_{b}
$$

If $a \in S_{j}, b \in S_{j+1}, j=1, \ldots, k-1$, and again $p \leqslant a, b \leqslant q$, then subtracting the equations of form (8) for these two cases gives us

$$
\sum_{i=p}^{q} \alpha_{i} \log \left(\alpha_{i} / \alpha\right)=c \log \left(\alpha_{b} / \alpha_{a}\right)
$$

Thus the quotients $\alpha_{a} / \alpha_{b}$ are constant for $a \in S_{j}, b \in S_{j+1}, j=1, \ldots, k-1$. These quotients are, of course, the slopes of $\tau$ over the intervals $I_{i}$ for $i \in S_{j}, j=1, \ldots, k-1$, $p \leqslant j \leqslant q$. Thus the maps corresponding to the critical points have constant slope over these intervals. It remains to show that this constant is equal to $\alpha / \alpha_{a}$, the slope of $\tau$ over intervals $I_{a}$ with $a \in S_{1}$.

Let $\beta_{i}$ equal the common value of $\alpha_{a}$ for $a \in S_{i}, p \leqslant a \leqslant q$. We must show that $\alpha / \beta_{i}=\beta_{1} / \beta_{2}$. Now we have shown that $\beta_{2} / \beta_{1}=\beta_{3} / \beta_{2}=\cdots=\beta_{k} / \beta_{k-1}$ and therefore $\beta_{i}=\beta_{2}^{i-1} / \beta_{1}^{i-2}, i=1, \ldots, k$. From (10) we have

$$
\sum_{i=p}^{q} \alpha_{i} \log \left(\alpha_{i} / \alpha\right)=c\left(\log \left(\beta_{2} / \beta_{1}\right)\right)
$$


which can be rewritten as

$$
\sum_{i=1}^{k} n_{i} \beta_{i} \log \left(\beta_{i} / \alpha\right)=\sum_{i=1}^{k} i n_{i} \beta_{i} \log \left(\beta_{2} / \beta_{1}\right)
$$

where $n_{i}$ equals the number of intervals $I_{a}$ with $p \leqslant a \leqslant q, a \in S_{i}$. Thus $\sum n_{i} \beta_{i}=\alpha$, and

$$
\sum_{i=1}^{k} n_{i} \beta_{i} \log \left(\frac{\beta_{2}^{i-1}}{\beta_{1}^{i-2}}\right)=\sum_{i=1}^{k} i_{i} \beta_{i} \log \left(\frac{\beta_{2}}{\beta_{1}}\right)
$$

or

$$
\sum_{i=1}^{k}\left(\text { in }_{i} \beta_{i}-(i-2) n_{i} \beta_{i}\right) \log \beta_{1}=\sum_{i=1}^{k}\left(\text { in }_{i} \beta_{i}-(i-1) n_{i} \beta_{i}\right) \log \beta_{2}+\sum_{i=1}^{k} n_{i} \beta_{i} \log \alpha .
$$

This yields

$$
2\left(\sum_{i=1}^{k} n_{i} \beta_{i}\right) \log \beta_{1}=\left(\sum_{i=1}^{k} n_{i} \beta_{i}\right) \log \beta_{2}+\left(\sum_{i=1}^{k} n_{i} \beta_{i}\right) \log \alpha
$$

Thus

$$
2 \alpha \log \beta_{1}=\alpha \log \beta_{2}+\alpha \log \alpha
$$

or $\beta_{1}^{2}=\beta_{2} \alpha, \alpha / \beta_{1}=\beta_{1} / \beta_{2}$.

We have shown that at the critical points the map $\tau$ must have constant slope over the intervals $I_{a}$, where $p \leqslant a \leqslant q$. On the other intervals the slope is arbitrary. Select these intervals so that the entire map has slope which is constant in absolute value, equal to $\lambda$, say. Since $A$ is irreducible we have shown in Corollary 1 that this is always possible and that $\lambda$ is the spectral radius of $A$. Thus the entropy of the absolutely continuous invariant measure for $\tau$ is maximal precisely when $\tau$ has slope $\pm \lambda$ over the intervals $I_{a}$ with $p \leqslant a \leqslant q$.

REMARKs. 1. If $p=1$ and $q=n$, then the absolutely continuous invariant measure is maximal if and only if $\tau$ has constant slope $\lambda$, that is, for maps of nonconstant slope the absolutely continuous invariant measure does not have maximal entropy.

2. If $p>1$ or $q<n$, there is a $(p-1)+(n-q)$ parameter family of maps (almost all of which have nonconstant slope), for which the absolutely continuous invariant measure has maximal entropy.

3. If $k=q-(p-1)$ is the number of entries in the block of 1's in $A$, then $n-k$ is the dimension of the space of piecewise linear Markov maps whose absolutely continuous invariant measure is maximal.

4. The examples of $\S 3$ are special cases of Theorem 3.

ACKNOWLeDgements. The authors are grateful to M. Scarowsky for helpful discussions.

\section{REFERENCES}

1. P. Collet and J. P. Eckmann, Iterated maps on the interval as dynamical systems, Birkhäuser, Boston, Mass., 1980.

2. T. Y. Li and J. A. Yorke, Period three implies chaos, Amer. Math. Monthly 82 (1975), 985-992.

3. R. May, Simple mathematical models with very complicated dynamics, Nature 261 (1976), 459-467. 
4. A. Lasota and J. A. Yorke, On the existence of invariant measures for piecewise monotonic transformations, Trans. Amer. Math. Soc. 183 (1973), 481-485.

5. R. Bowen, Invariant measures for Markov maps of the interval, Comm. Math. Phys. 69 (1979), 1-17.

6. M. Misieurewicz, Absolutely continuous measures for certain maps of an interval, Publ. Math. I.H.E.S., Paris, 1980.

7. G. Pianigiani, Absolutely continuous invariant measures for the process $X_{n+1}=A X_{n}\left(1-X_{n}\right)$, Boll. Un. Mat. Ital. 16A (1979), 374-378.

8. R. L. Adler, F-expansions revisited, Lectures Notes in Math., vol. 318, Springer, Berlin and New York, 1973, pp. 1-5.

9. M. Misiurewicz, Invariant measures for continuous transformation of $[0,1]$ with zero topological entropy, Lecture Notes in Math., vol. 729, Springer, Berlin and New York, 1980, pp. 144-152.

10. F. Hofbauer, $\beta$-shifts have unique maximal measure, Monatsh. Math. 85 (1978), 189-198.

11. , Maximal measures for piecewise monotonically increasing functions on [0,1], Lecture Notes in Math., vol. 729, Springer, Berlin and New York, 1979, pp. 66-77.

12. M. Misiurewicz, Absolutely continuous measures for certain Markov maps of an interval, I.H.E.S., Paris, 1979.

13. R. L. Adler, A. C. Konheim and W. H. McAndrew, Topological entropy, Trans. Amer. Math. Soc. 114 (1965), 309-319.

14. A. Boyarsky and M. Scarowsky, On a class of transformations which have unique absolutely continuous invariant measures, Trans. Amer. Math. Soc. 255 (1979), 243-262.

15. N. Friedman and A. Boyarsky, Matrices and eigenfunctions induced by Markov maps, Linear Algebra Appl. 38 (1981), 141-147.

16. A. Boyarsky and G. Haddad, All invariant densities of piecewise linear Markov maps are piecewise constant, Adv. Appl. Math. 2 (1981), 284-289.

17. E. Senenta, Non-negative matrices and Markov chains, 2nd ed., Springer-Verlag, Berlin and New York, 1981.

18. P. Billingsley, Ergodic theory and information, Wiley, New York, 1965.

19. P. Walters, An introduction to ergodic theory, Springer-Verlag, Berlin and New York, 1982.

20. P. Lancaster, Theory of matrices, Academic Press, New York, 1969.

21. Z. Nitecki, Differentiable dynamics, M.I.T. Press, Cambridge, Mass., 1971.

22. W. Parry, Intrinsic Markov chains, Trans. Amer. Math. Soc. 112 (1969), 55-66.

23. Symbolic dynamics and transformations of the unit interval, Trans. Amer. Math. Soc. 122 (1966), 368-378.

Department of Mathematics, loyola Campus, Concordia University, Montreal, Canada H4B 1R6 